difficulties. What is most bewildering for the fitter is the complete absence of suction, with the lens actually falling out of the eye as the upper lid is lifted. As regards the patient, the optical effect of peripheral air bubbles is hardly perceptible and, if so, easily overcome, but holes showing in the palpebral aperture are not aesthetic although a perforated contact lens in a comfortable white eye is much less conspicuous than an unperforated one in an irritable eye however slight the irritation that precedes and accompanies the veil.

However, once there is a working principle, I am confident that ways will be found, perhaps through a combination of air pockets and channels, and holes where they are not visible (porous substances would probably have a limited delaying action only, like buffer solutions), to overcome this last minor trouble with contact lenses.

I would like to take this opportunity of thanking Mr. F. A. Williamson-Noble for his kindness in allowing me to make use of material from his hitherto unpublished experiments. For permission to refer to their cases I wish to acknowledge my indebtedness to Lady Duke-Elder, Mr. A. Rugg-Gunn, Mr. P. G. Doyne and Mr. T. G. Wynne Parry, and in particular to Professor Ida Mann for her invaluable co-operation over many years.

Technical facilities were generously placed at my disposal by Messrs. Theodore Hamblin, Ltd.

\title{
CONJUNCTIVAL FINDINGS IN CASES OF COLD HAEMAGGLUTINATION*
}

\author{
BY \\ SÜREYYA GÖRDÜREN \\ ANKARA
}

THE investigation of conjunctival blood circulation in cases of cold haemagglutination, where capillary and blood changes occur, is very important.

Before the following details I will explain some peculiarities of conjunctival blood circulation. Conjunctival vessels contrasting with the bright colour of scleral tunic can be clearly seen with the naked eye. When we use slit-lamp microscopy it becomes the ideal place for capillaroscopy.

* Received for publication, April 11, 1946. 
The conjunctiva has two kinds of vessels, one superficial and, somewhat lighter in colour, the other deep and of a dark blue colour. Especially in superficial vessels one can examine in detail the vasomotor reactions, the stream of blood cells.

Recently we had the opportunity of having these examinations on two patients suffering from cold haemagglutination.

1-Ayvaz Alçin, aged 39 years, Kalecik.

2-Hüseyin Evren, aged 22 years, Tokat.

Before the experiment we made surface anaesthesia by instilling 3 per cent. cocaine solution into the conjunctival sac. The least eye movements during the slit-lamp examination of conjunctival vessels, especially with high magnitude, change the eye-field and spoil a thorough investigation. This is the reason why we used cocaine, although it had some interference with the vessel walls.

For cooling the conjunctival sac we used isotonic salt solution in varying temperatures. The results may be summarised as follows :-

TABLE I

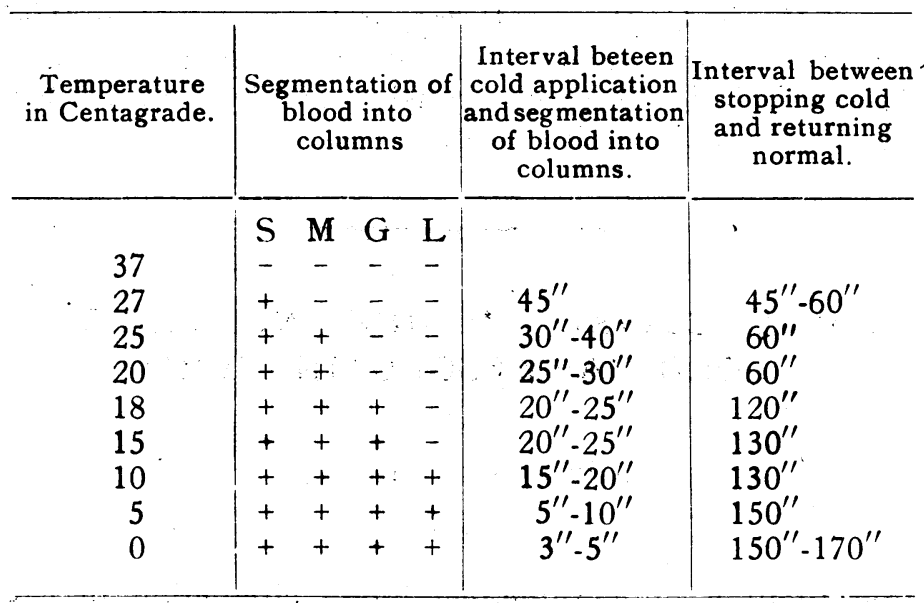

$\mathrm{S}=$ superficial vessels, $\mathrm{M}=$ medium sized vessels, $\mathrm{G}=$ greater vessels, $\mathrm{L}=$ large vessels.

A routine examination of the above table shows the following results :-

1. In body temperature $\left(37^{\circ}\right)$ the blood column has the normal appearance in vessels. Under this temperature this normal appearance began to disappear and at $27^{\circ}$ the changes became 


\section{Conjunctival Findings in Cases of Cold HAEMAGGLUTINATION}

obvious, blood corpuscles agglutinate in vessels, and divide into columns. With the influence of cold first the blood stream in all conjunctival vessels increases, and in some where previously it was not obvious it becomes visible. This can be easily understood; in the normal lumen of the vessels the blood corpuscles have a slow and scattered course. When vaso-constriction occurs they close up and fill the narrow lumen and have a swift course and are thus rendered obvious and visible.

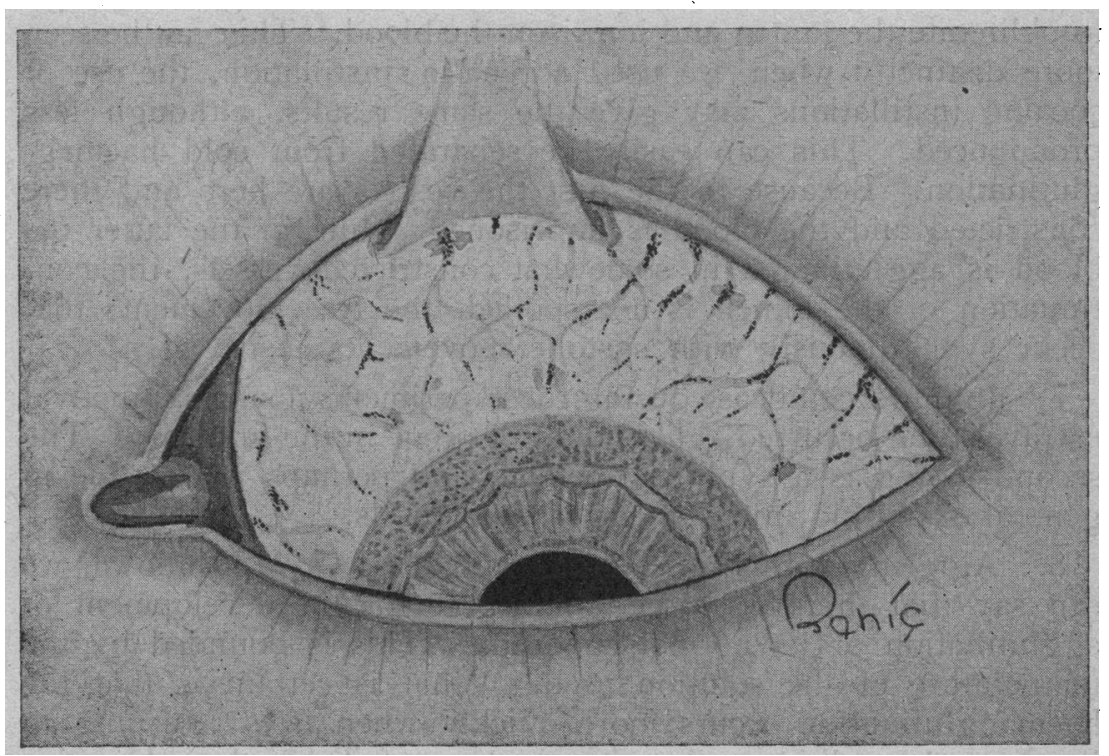

2. The segmentation and division of the blood into columns occurs first in the superficial small vessels. With the decrease in temperature the same procedure occurs in the medium sized, in the great and in the greater vessels. This can be explained by the fact that the small and superficial vessels are first affected by the cold. Here the vaso-constriction effect is greater and this is added to haemagglutination.

3 . In the arteries where the blood columns have been divided into pieces, a movement synchronous with the systolic movements persists a certain time, which changes with the temperature of the solution. Then the blood stream stops and takes on the appearance of thin red rods.

When the temperature is rather high this occurs in the small and superficial vessels only, when it falls the great vessels are also affected. 
4. When the segmented blood columns are examined with high magnification their borders are irregular and have the appearance of a flocculation made of aggregated blood cells.

5. When the temperature decreases the length of columns decreases, and when the temperature increases the length of columns also increases.

6: 'We had control tests on ten healthy men. Here cold caused a vaso-constriction and this caused the cessation of the blood stream, here and there the constriction was great enough to obliterate the lumen and imprison the blood. This can be seen more distinctly when we used adrenalin instillation, the use of cocaine instillations may give the same results, although less pronounced. This can easily be separated from cold haemagglutination. Because in the first the vessels are here and there constricted and the blood is imprisoned, while in the latter the blood is agglutinated in somewhat constricted vessels; the continuation of the lumen is not spoiled, the free movements that occur synchronously with systolic movements justify it.

7. In our second case during the experiments a subconjunctival extravasation occurred. This did not occur in the first case. The second case was a syphilitic person, and perhaps it was due to general syphilitic involvement of the vessels.

8. Agreeing with Daniel States and Jesse G. M. Bullowa, we can say that there is no certain degree for the development of agglutination or its normal condition. This is bounded by the temperature of the solution used. What is certain is that the haemagglutination occurs more quickly when it is returning to its normal condition. An examination of Table I makes this clear. There is one more point, the figures in the table are not absolute. Hence we used a minimum and a maximum time. The reason is clear. We cannot find the same mathematical results in all vessels of the conjunctiva. Even the normal conjunctival vessels show capricious changes. One cannot give absolute figures for these vessels which show capricious changes even under normal conditions. On the other hand, according to nutritional requirements, in some conjunctival vessels the blood stream may stop for a varying period and may start again, and this renders the mechanism more capricious. 